\title{
A rule-based parameter aided with object-based classification approach for extraction of building and roads from WorldView-2 images
}

\begin{abstract}
Roads and buildings constitute a significant proportion of urban areas. Considerable amount of research has been done on the road and building extraction from remotely sensed imagery. However, a few of them have been concentrating on using only spectral information. This study presents a comparison between three object-based models for urban features' classification, specifically roads and buildings, from WorldView-2 satellite imagery. The three applied algorithms are support vector machines (SVMs), nearest neighbour (NN) and proposed rule-based system. The results indicated that the proposed rules in this study, despite the spectral complexity of land cover types, performed a satisfactory output with an overall accuracy of $92.92 \%$. The advantages offered by the proposed rules were not provided by other two applied algorithms and it revealed the highest accuracy compared to SVM and NN. The overall accuracy for SVM was $76.76 \%$, which is almost similar to the result achieved by NN (77.3\%).
\end{abstract}

Keyword: Building extraction; Road; Remote sensing; SVM; NN; Rule-based; WorldView2 images 